\title{
VALIDEZ Y REPRODUCIBILIDAD DEL SISTEMA DE PUNTUACIÓN DE NUGENT PARA EL DIAGNÓSTICO DE VAGINOSIS BACTERIANA EN MUJERES EMBARAZADAS*
}

\author{
Lina María Vera C. ${ }^{1,2, a}$, Nahyr López B. ${ }^{1, b}$, Alba Lucía Arámbula. 3,c \\ 1 Grupo GUINDESS, Departamento de Salud Pública, Escuela de Medicina, Universidad Industrial de Santander, \\ Bucaramanga, Colombia. ${ }^{2}$ Department of Population Health Sciences, University of Wisconsin at Madison, WI, USA. \\ 3 Escuela de Bacteriología y Laboratorio Clínico, Universidad Industrial de Santander, Colombia.
}

a Médico Cirujano, magíster en Epidemiología. ${ }^{b}$ Ingeniera de Sistemas, magíster en Bioestadística. ${ }^{\mathrm{C}}$ Bacterióloga, magíster en Microbiología.

* Investigación financiada por la Universidad Industrial de Santander.

\section{RESUMEN}

Antecedentes: La vaginosis bacteriana se encuentra entre 15 a $20 \%$ de las mujeres embarazadas y el método rutinario para diagnosticarla es el estudio del flujo vaginal aplicando los criterios de Amsel. Sin embargo, existen otros métodos diagnósticos como el sistema de Nugent, que ha mostrado adecuada validez a menor costo. Objetivo: Evaluar la validez y reproducibilidad del sistema de Nugent para el diagnóstico de vaginosis bacteriana en embarazadas. Método: Estudio de evaluación de pruebas diagnósticas. Se estudiaron 100 mujeres con embarazo de bajo riesgo. A cada paciente se le tomaron dos muestras de secreción de flujo vaginal y tres evaluadores examinaron cada muestra. Se evaluó la validez de los criterios de Nugent comparándolos con los de Amsel (prueba de oro) y se obtuvo el mejor punto de corte usando la curva del receptor-operador (ROC), para calcular la sensibilidad, especificidad y valores predictivos. La reproducibilidad se midió con el coeficiente Kappa. Resultados: El promedio de edad fue 25,6 años y edad gestacional 23 semanas. Un 19\% había tenido infección vaginal previamente. La prevalencia actual de vaginosis bacteriana fue 16\% (IC95\%: 9,43\%; 24,67\%). El área bajo la curva ROC fue 0,98 (IC95\%: 0,95; 1,00). La sensibilidad fue 1,00 (IC95\%: 0,77; 1,00), especificidad 0,96 (IC95\%: 0,90; 0,99), valor predictivo positivo 0,82 (IC95\%: 0,57; 0,96) y valor predictivo negativo 1,00 (IC95\%: 0,95; 1,00). Conclusión: El sistema de Nugent para el diagnóstico de vaginosis bacteriana en embarazadas es una prueba válida y reproducible para implementar rutinariamente durante el control prenatal.

\section{PALABRAS CLAVE: Vaginosis bacteriana, validez diagnóstica, reproducibilidad, prueba de Nugent}

\section{SUMMARY}

Background: The bacterial vaginosis is found in $15 \%$ to $20 \%$ of the pregnant women and the study of the vaginal discharge using the Amsel criteria is the usual method to diagnose it. However, there are other diagnostic methods like the Nugent Score System which has shown to be accurate and less expensive. Objective: To evaluate the accuracy and reliability of the Nugent Score System for diagnosing bacterial vaginosis in pregnant women. Methods: This is a study for evaluating medical tests. We studied 100 women who had a low risk pregnancy. Two samples of the vaginal discharge were taken in each woman and three masked 
evaluators examined each sample. The accuracy of the Nugent Score System was evaluated compare it with the Amsel criteria as a gold standard. To calculate the sensibility, specificity, positive predictive value, and negative predictive value of the Nugent Score System, we got the best cut point from the Nugent Score System using the receiver operator curve (ROC). The reliability was measured using the Kappa coefficient. Results: The average age was 25.6 years and the average gestational age was 23 weeks. A $19 \%$ of the women had had a vaginal infection previously. The current prevalence of bacterial vaginosis was $16 \%$ (95\%Cl: $9.43 \%$; $24.67 \%)$. The ROC analysis showed an area under the curve of 0.98 (95\%Cl: $0.95 ; 1.00)$. The sensitivity was $1.00(95 \% \mathrm{Cl}: 0.77 ; 1.00)$, the specificity $0.96(95 \% \mathrm{Cl}: 0.90 ; 0.99)$, the positive predictive value $0.82(95 \% \mathrm{Cl}: 0.57 ; 0.96)$, and the negative predictive value $1.00(95 \% \mathrm{Cl} 0.95 ; 1.00)$. Conclusion: The Nugent Score System for diagnosing bacterial vaginosis in pregnant women is a valid and reproducible test for being implemented routinely during the prenatal care.

\section{KEY WORDS: Bacterial vaginosis, diagnostic accuracy, reliability, Nugent test}

\section{INTRODUCCIÓN}

Las infecciones vaginales constituyen una patología frecuente en todos los grupos de edad pero afectan principalmente a mujeres en edad fértil y con vida sexual activa. En Colombia se notifican anualmente más de 70.000 casos nuevos de infecciones de transmisión sexual (ITS) dentro de las cuales se encuentra el síndrome de secreción vaginal que incluye la presencia de vaginosis bacteriana (VB) con una prevalencia del $29,1 \%$, seguida de la infección por Cándida spp (22,8\%), Chlamydia trachomatis (3,7\%), Treponema pallidum $(3,7 \%)$ y Trichomonas vaginalis $(1,1 \%)$ (1).

La prevalencia de VB va de $10 \%$ en mujeres asintomáticas embarazadas, a $50 \%$ en mujeres que consultan por alguna afección de la secreción anormal del flujo vaginal (2-5). La VB es responsable de una considerable morbilidad entre mujeres en edad reproductiva y está asociada con complicaciones obstétricas que incluyen parto de pretérmino, ruptura prematura de membranas, corioamnionitis, aborto espontáneo, infección poscesárea y bajo peso al nacer $(2,3,6)$. Estas complicaciones gestacionales generan una elevada morbimortalidad perinatal que sería evitable, si se llevara a cabo un adecuado control prenatal, con intervenciones impacto demostrado como lo son el diagnóstico y manejo oportuno de la bacteriuria asintomática y la vaginosis bacteriana $(7)$.

Actualmente, el método considerado como estándar de oro para hacer el diagnóstico de VB y de uso común en países en vías de desarrollo, es el estudio del flujo vaginal aplicando los criterios de Amsel $(8,9)$. Este método requiere del uso de reactivos con costo adicional al del recurso humano, que puede dificultar su utilización rutinaria en poblaciones económicamente deprimidas. El diag- nóstico de VB, basado en la coloración de Gram del flujo vaginal ha demostrado ser un método de alta confiabilidad (8-10,11-14), con una muy buena reproducibilidad y validez $(8,9,13,15)$, rápido y con una mejor relación costo-beneficio $(9,12,16)$, siempre y cuando esté estandarizado $(8,9,17)$.

Nugent y cols (8), hicieron de la coloración de Gram del flujo vaginal un método objetivo y estandarizado que evalúa la biota de la vagina. Diseñaron un sistema de puntos (cero a diez) basado en la suma ponderada de los siguientes morfotipos bacterianos: bacilos Gram positivos tipo Lactobacillus, los cocobacilos Gram variables pleomórficos tipo Gardnerella/Bacteroides y los bacilos Gram negativos curvos tipo Mobiluncus spp, debido a su gran reproducibilidad intercentro.

El objetivo de este estudio fue evaluar la validez y reproducibilidad del sistema de puntuación de Nugent para el diagnóstico de vaginosis bacteriana en embarazadas.

\section{MATERIALES Y MÉTODOS}

Este estudio se ajustó a las normas internacionales para investigación en seres humanos y fue aprobado por el comité de ética de la Facultad de Salud de la Universidad Industrial de Santander. Diseño del estudio: Estudio de evaluación de pruebas diagnósticas con muestreo naturalístico o de corte transversal.

Población de estudio: Embarazadas asistentes al programa de control prenatal en dos instituciones de salud.

Tamaño de la muestra: Se determinó siguiendo las recomendaciones de Chmura (19) para calcular la validez de las pruebas diagnósticas. Se trabajó con una prevalencia de VB del 15 a $20 \%$ en mujeres embarazadas (2). Se evaluaron en total 
100 mujeres embarazadas asistentes al programa de control prenatal.

Criterios de inclusión y exclusión: Se incluyeron mujeres con prueba de embarazo en sangre positiva, asistentes al programa de control prenatal de las instituciones participantes, residentes en Bucaramanga y el área metropolitana, que aceptaron participar en el estudio. Se excluyeron las mujeres con embarazos clasificados por el médico ginecoobstetra como de alto riesgo.

Variables del estudio: Se evaluaron variables sociodemográficas como edad, procedencia, estado civil, escolaridad y ocupación. Factores de riesgo para VB como uso de duchas vaginales, edad de inicio de actividad sexual, múltiples compañeros sexuales, uso de métodos de anticoncepción y tabaquismo. Se incluye edad gestacional, antecedentes patológicos e historia de tratamiento farmacológico reciente.

Criterios de Amsel: Consiste en evidenciar la presencia de una descarga vaginal homogénea, test de aminas positivo, $\mathrm{pH}$ vaginal $\geq 4,5$ y presencia de células guía bajo la observación del fluido vaginal examinado al microscopio (20). El resultado de la aplicación de los criterios de Amsel puede ser positivo para diagnóstico de VB que corresponde a quienes obtienen positivos al menos tres de los cuatro criterios o negativo en quienes tienen menos de tres criterios positivos.

Prueba de Nugent: Se hace mediante coloración de Gram del flujo vaginal y se evalúan los morfotipos presentes en la muestra (Tabla I). La interpretación propuesta para la escala es de 0-3 puntos normal, de 4-6 puntos se considera intermedio (debiéndose repetir la prueba una semana después) y de 7-10 puntos es diagnóstico de VB.

\section{Tabla I}

\section{PONDERACIÓN DEL SISTEMA DE} PUNTUACIÓN DE NUGENT $(5,8)$

\begin{tabular}{cccc}
\hline & \multicolumn{3}{c}{ Morfotipo* $^{*}$ Ponderación } \\
\cline { 2 - 4 } & Lactobacillus & Mobiluncus & $\begin{array}{c}\text { Gardnerella/ } \\
\text { Bacteroides }\end{array}$ \\
\hline 0 & $>30$ & 0 & 0 \\
1 & $5-30$ & $1-4$ & $<1$ \\
2 & $1-4$ & $>5$ & $1-4$ \\
3 & $<1$ & & $5-30$ \\
4 & 0 & & $>30$ \\
\hline
\end{tabular}

* Promedio de microorganismos/campo observado en aceite de inmersión con objetivo 100x.
Procedimientos de recolección de la información: A todas las pacientes se les dio información sobre el estudio, se les pidió su consentimiento para participar y se citaron a la toma de muestra. En la toma de muestra, se les solicitó que firmaran el consentimiento informado y se procedió a recolectar la información y a realizar el examen.

Toma de muestra y procesamiento: Mediante especuloscopia directa se hizo una observación de las características de la cavidad vaginal y del cérvix y se tomaron dos muestras, con escobillones diferentes, de la secreción presente en las paredes vaginales. La primera muestra se depositó en un tubo de ensayo para ser mezclada con $1 \mathrm{ml}$ de solución salina al $0,9 \%$, se hizo un montaje húmedo que se observó al microscopio con el objetivo de 40x para buscar células guía. La segunda muestra fue distribuida inmediatamente en forma de ovillo sobre la lámina portaobjetos, se dejó secar al aire y después se fijó con calor seco para finalmente colorearla con la tinción de Gram. Al momento de hacer la toma de muestra se asignó un código a cada paciente para su identificación. Posteriormente y de manera secreta, cada muestra fue identificada con un segundo código. El evaluador 1 procesó y leyó las muestras que eran tomadas, realizó la prueba de Amsel y reportó los resultados en un formato usando el primer código asignado a cada muestra como identificador de la misma. Los otros dos evaluadores participantes en el estudio realizaron la primera lectura de la prueba de Nugent a las muestras que fueron tomadas, guardando la independencia en sus lecturas. Los resultados se reportaron en otro formato usando nuevamente el primer código asignado a cada muestra. Finalmente, las 100 muestras fueron rotuladas (por uno de los investigadores no evaluadores) con el segundo código asignado a la muestra y leídas nuevamente por cada uno de los tres evaluadores aplicando los criterios de Nugent. Los tres profesionales que realizaron esta evaluación fueron entrenados en la aplicación de las pruebas, no conocieron la historia clínica de la paciente y estuvieron enmascarados al resultado de las pruebas durante todo el estudio.

Control de calidad de la información: Las muestras fueron custodiadas diariamente por uno de los investigadores (no evaluador) y para el tránsito de las muestras por los diferentes evaluadores se llevó un registro en un formato de chequeo que incluía los códigos de las muestras. En el diseño de la base de datos se establecieron controles para la captura de la información y se realizó doble digitación de cada uno de los formularios de registro. A los archivos definitivos se les realizó un proceso de depuración donde se detectaron algunas inconsistencias de 
datos, que fueron corregidas antes de producir los resultados del estudio.

Procesamiento y análisis de los datos: La captura de la información se realizó utilizando el programa Epi-info 6.04d (21) y para el procesamiento de los datos se utilizó el software Stata 8.0 (22). Se realizó un análisis univariado para describir cada una de las variables del estudio utilizando medidas de tendencia central y variabilidad para las variables continuas y proporciones para las variables discretas. Se evaluó la validez del sistema de puntuación de Nugent comparándolo con el estándar de oro (Criterios de Amsel). Se realizó el análisis del puntaje de Nugent mediante la curva ROC para determinar el mejor punto de corte para el diagnóstico de vaginosis bacteriana en mujeres embarazadas. Se calcularon la sensibilidad, especificidad y valores predictivos del sistema de puntuación de Nugent de acuerdo con el mejor punto de corte obtenido en la curva ROC. Para todos los análisis se siguieron las recomendaciones de Chmura (19) para el muestreo realizado. La reproducibilidad intraobservador e interobservador del sistema de puntuación de Nugent para el diagnóstico de vaginosis bacteriana en mujeres embarazadas se midió con el índice de Kappa. También se realizó un análisis para identificar los factores de riesgo asociados a la VB.

\section{RESULTADOS}

Descripción de la población: Las edades de las 100 participantes fluctuaron entre 15 y 42 años, siendo el promedio de 25,6 años $\pm 6,2$ años. El $62,6 \%$ pertenecía a estratos socioeconómicos 1 y 2 , el $37,4 \%$ a estratos 3 y 4 . El $82 \%$ era amas de casa, $9 \%$ comerciante, $5 \%$ estudiante y $4 \%$ tenía trabajo de servicios. El promedio de semanas de gestación fue de $22,8 \pm 8,4$ semanas (rango: 4 y 36 semanas). El 49\% tenía unión libre, el $34 \%$ casada, 16\% soltera y una divorciada. El nivel de educación era $56 \%$ secundaria, $18 \%$ primaria, $13 \%$ nivel técnico y $13 \%$ universitario.

En cuanto a los factores de riesgo para VB se encontró que solo dos mujeres reportaron haber fumado durante su embarazo, 2 y 3 cigarrillos al día respectivamente. El $68 \%$ de las participantes había usado alguna vez métodos anticonceptivos, siendo las píldoras el de mayor uso. El $82 \%$ de las mujeres dijo haber tenido un compañero sexual en el último mes, $17 \%$ no tuvo y solo una mujer tuvo dos compañeros sexuales en el mismo tiempo. El antecedente de infección vaginal fue reportado por el $19 \%$ de las participantes y las más frecuentes fueron vagino- sis bacteriana $(5 \%)$, candidiasis $(12 \%)$, sífilis $(1 \%)$ y herpes genital (1\%). Las duchas vaginales fueron usadas durante el embarazo por 4 mujeres y en el momento de la encuesta el 59\% estaba tomando algún medicamento.

Prevalencia de VB: El 16\% de las mujeres presentó resultado positivo para la prueba de VB según los criterios de Amsel (IC95\%: 9,43\%; 24,67\%), en correspondencia con el hallazgo según la prueba de Nugent, en la que el valor correspondió al 14,43\% de 97 mujeres que fueron clasificadas (IC95\%: $8,12 \% ; 23,02 \%)$. El frotis vaginal de 3 mujeres, aplicando el sistema de Nugent fue declarado "no concluyente". Aquellas mujeres que pertenecen a estrato 1 o 2 tuvieron 5,1 veces más riesgo de presentar VB que aquellas de estratos superiores $(p<0,0247)$. Otros factores como el estado civil, el nivel de estudios, la ocupación, haber usado alguna vez métodos anticonceptivos, las semanas de gestación, haber tenido compañeros sexuales en el último mes, haber presentado anteriormente algún tipo de infección vaginal y estar tomando algún medicamento al momento de la encuesta no se relacionaron con la presencia de VB.

Validez del sistema de puntuación de Nugent: Analizando la valoración de la prueba de Nugent en su escala continua, que fluctúa entre 0 y 10 , se construyó la curva ROC para identificar los posibles valores de corte que mostraran diferentes niveles de discriminación de la prueba, comparado con el resultado del estándar de oro. Se encontró un área bajo la curva de $97,54 \%$ (IC95\%: 94,68\%; 100\%), con error estándar de 0,0146 (Figura 1). El mejor punto de corte correspondió a 5 o más, y se usó para calcular la sensibilidad, especificidad y valores predictivos que se muestran en la Tabla II.

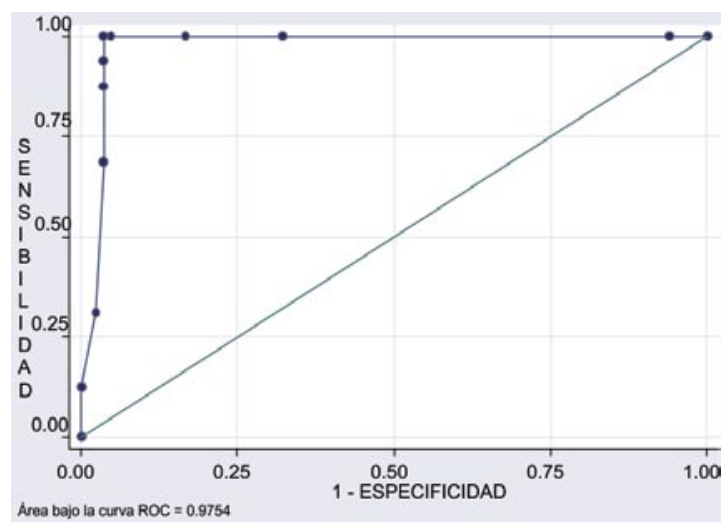

Figura 1. Curva ROC del análisis del sistema de puntuación de Nugent. 
Tabla II

\section{SENSIBILIDAD, ESPECIFICIDAD Y VALORES PREDICTIVOS DEL SISTEMA DE PUNTUACIÓN DE NUGENT}

\begin{tabular}{lcc}
\hline Resultado & Valor & IC 95\% \\
\hline Sensibilidad & 1,0000 & 0,$7683 ; 1,0000$ \\
Especificidad & 0,9639 & 0,$8979 ; 0,9924$ \\
Valor predictivo positivo & 0,8235 & 0,$5656 ; 0,9620$ \\
Valor predictivo negativo & 1,0000 & 0,$9549 ; 1,0000$ \\
\hline
\end{tabular}

Reproducibilidad del sistema de puntuación de $\mathrm{Nu}$ gent: La reproducibilidad intraobservador reportó porcentajes de concordancia de $97 \%$ y $84 \%$ para los evaluadores 1 y 2 , que fueron quienes repitieron la prueba de Nugent, con valores Kappa de 0,90 y 0,63 respectivamente (estadísticamente significativos). La reproducibilidad interobservador entre los evaluadores 1 y 2 , arrojó un $88 \%$ de acuerdos, con valor Kappa de 0,68, y entre los evaluadores 1 y 3 , los acuerdos fueron del $94 \%$ con valor Kappa de 0,82 , mientras que entre los evaluadores 2 y 3 , las concordancias correspondieron a un $89 \%$ con valor Kappa de 0,72. Todos estos resultados fueron estadísticamente significativos $(p<0,05)$.

\section{DISCUSIÓN}

La VB es una infección ampliamente estudiada y es especialmente importante en las embarazadas debido a su asociación con complicaciones obstétricas $(2,3,6)$. Si bien las mujeres embarazadas tienen mayor susceptibilidad a este tipo de infecciones debido a los cambios que ocurren durante el embarazo en la flora vaginal, la población estudiada tuvo en general una baja proporción de factores de riesgo para vaginosis bacteriana.

El método más ampliamente usado y aceptado para el diagnóstico de vaginosis bacteriana tanto en mujeres embarazadas como no embarazadas es el sistema de puntuación de Nugent (23). Sin embargo, en Colombia se siguen aplicando los criterios de Amsel para hacer este diagnóstico y no hay evidencia de la utilidad del uso del sistema de puntuación de Nugent en la población general ni en embarazadas que se beneficiarían de la implementación de un método válido y que requiere menos insumos y en consecuencia menos costos para su ejecución. Además de la reducción promedio de $\$ 1000$ por paciente, en los costos del proceso diagnóstico con el uso de la prueba de Nugent, las pacientes se beneficiarían de la realización de un examen más cómodo, puesto que no se requiere el uso de espéculo para obtener la muestra adecuada para la aplicación del sistema de puntuación de Nugent.

La validez diagnóstica de una prueba puede variar dependiendo del método, el examinador, la técnica usada y la prevalencia de la condición en la población (19). El sistema de puntuación de Nugent es una prueba diagnóstica para la VB que ha sido validada y utilizada como prueba de oro en otras poblaciones $(10,14,23-28)$. La decisión de implementar este sistema diagnóstico como prueba de rutina en las mujeres embarazadas de nuestro país debe ser sustentada en evidencia científica sobre su utilidad en nuestra población.

En este estudio utilizamos el análisis ROC para determinar el mejor punto de corte del sistema de puntuación de Nugent el cual correspondió a un valor de 5 o más qué es diferente al punto de corte tradicionalmente usado en otros estudios $(10,14,23$ 28). Sin embargo, la sensibilidad, especificidad y valores predictivos encontrados en este estudio fueron superiores a los reportados previamente en esos estudios.

\section{CONCLUSIÓN}

Los resultados de este estudio indican que una vez estandarizada la prueba y se hace una adecuada capacitación de los evaluadores, el sistema de puntuación de Nugent tiene buena validez, excelente capacidad diagnóstica y altamente reproducible para el diagnóstico de vaginosis bacteriana en embarazadas colombianas.

\section{BIBLIOGRAFÍA}

1. Instituto Nacional de Salud, Ministerio de Salud de Colombia. Situación de las Enfermedades de Transmisión Sexual, Colombia, 1976-2000. Inf Quinc Epidemiol Nac 2001;6(22):329-44.

2. Angel E, Ruiz Al. Repercusiones perinatales de las infecciones cervicovaginales. Rev Fac Med UN Col 1995;43(1):21-5.

3. Morris M, Nicoll A, Simms I, Wilson J, Catchpole M. Bacterial vaginosis: a public health review. Br J Obstet Gynaecol 2001;108:439-50.

4. Yen S, Shafer M, Moncada J, Campbell C, Flinn S, Boyer C. Bacterial vaginosis in sexually experienced non-sexually experienced young women entering the military. Obstet Gynecol 2003;102:927-33.

5. Rado A, Mere J, García M. Riesgo de las complicaciones de vaginosis bacteriana en gestantes. Ginecol Obstet (Perú) 2001;47(3):177-183.

6. Hillier SL, Nugent RP, Eschenbach DA, et al. As- 
sociation between bacterial vaginosis and preterm delivery of a low-birth-weight infant. $\mathrm{N}$ Engl $\mathrm{J}$ Med 1995;333:1737-42.

7. Ortiz El. Estrategias para la prevención del bajo peso al nacer en una población de alto riesgo, según la medicina basada en la evidencia. Colomb Med 2001;32:159-62.

8. Nugent RP, Krohn MA, Hillier SL. Reliability of diagnosis bacterial vaginosis is improved by a standardized method of gram stain interpretation. J Clin Microbiol 1991;29(2):297-301.

9. Zarakolu P, Hodoglugil NNS, Aydin F, Tosun I, Gozalan A, Unal S. Reliability of interpretation of gramstained vaginal smears by nugent's scoring system for diagnosis of bacterial vaginosis. Diagn Microbiol Infect Dis 2004; $48: 77-80$.

10. Schwebke JR, Hillier SL, Sobel JD, McGregor JA, Sweet RL. Validity of the vaginal gram stain for the diagnosis of bacterial vaginosis. Obstet Gynecol 1996;88(4Pt 1):573-6.

11. Spiegel CA, Amsel R, Holmes KK. Diagnosis of bacterial vaginosis by direct gram stain of vaginal fluid. $J$ Clin Microbiol 1983;18(1):170-7.

12. Krohn MA, Hillier SL, Eschenbach DA. Comparison of methods for diagnosis bacterial vaginosis among pregnant women. J Clin Microbiol 1989;27(6):126671.

13. Mazzulli T, Simor AE, Low DE. Reproducibility of interpretation of gram-stained vaginal smears for the diagnosis of bacterial vaginosis. J Clin Microbiol 1990;28(7):1506-8.

14. Gratacós E, Figueras F, Barranco M, Ros R, Andreu A, Alonso PL. Prevalence of bacterial vaginosis and correlation of clinical to Gram stain diagnostic criteria in low risk pregnant women. Europ $\mathrm{J}$ Epidemiol 1999;15(10):913-6.

15. Nelson DB, Bellamy S, Gray TS, Nachamkin I. Self-collected versus provider-collected vaginal swabs for the diagnosis of bacterial vaginosis: an assessment of validity and reliability. J Clin Epidemiol 2003;56(9):8626.

16. Tam MT, Yungbluth M, Myles T. Gram stain method shows better sensitivity than clinical criteria for detection of bacterial vaginosis in surveillance of pregnant, low-income women in a clinical setting. Infect Dis Obstet Gynecol 1998;6(5):204-8.
17. Larsson PG, Carlsson B, Fahraeus L, Jakobsson $T$, Forsum U. Diagnosis of bacterial vaginosis: need for validation of microscopic image area used for scoring bacterial morphotypes. Sex Transm Infect 2004;80:637.

18. World Medical Association. Declaration of Helsinki: ethical principles for medical research involving human subjects. Helsinki, Finlandia: WMA, 1964.

19. Chmura H. Evaluating medical tests: objective and quantitative guidelines. 1 ed. Newbury Park, Estados Unidos: Sage Publications, 1992. 342 p.

20. Amsel R, Totten PA, Spiegel CA, Chen KC, Eschenbach D, Holmes KK. Nonspecific vaginitis. Diagnostic criteria and microbial and epidemiologic associations. Am J Med 1983;74(1):14-22.

21. CDC/WHO. Epi Info 6.04d. [Fecha de acceso: 1 noviembre de 2003]. URL disponible en: http://www.cdc. gov/epiinfo

22. StataCorp. Stata Statistical Software: Release 8.0. College Station, TX: Stat Corporation; 2002.

23. Culhane J, Desanto D, Goldenberg R, McCollum K, King F, Guaschino S. Variation in Nugent score and leukocyte count in fluid collected from different vaginal sites. Obstet Gynecol 2005;105:120-3.

24. Myziuk L, Romanowski B, Johnson S. BV blue test for diagnosis of bacterial vaginosis. J Clin Microbiol 2003;41(5):1925-8.

25. West B, Morison L, Van Der Loeff M, Gooding E, Awasana A, Demba E, Mayaud P. Evaluation of a new rapid diagnostic kit (FemExam) for bacterial vaginosis in patients with vaginal discharge syndrome in The Gambia. Sex Trans Dis 2003;30(6):483-9.

26. Sha B, Chen H, Wang Q, Reza M, Cohen M, Spear G. Utility of Amsel criteria, Nugent score, and quantitative PCR for Gardnerella vaginalis, Mycoplasma hominis, and Lactobacillus spp. for diagnosis of bacterial vaginosis in human inmunodeficiency-virus infected women. J Clin Microbiol 2005;43(9):4607-4612.

27. Hogan V, Culhane J, Hitti J, Rauh V, McCollum K, Agnew K. Relative performance of three methods for diagnosing bacterial vaginosis during pregnancy. Matern Child Health J 2007;11:532-9.

28. Eriksson K, Forsum U, Bjornerem A, Platz-Christensen J, Larsson P. Validation of the use of Pap-stained vaginal smears for diagnosis of bacterial vaginosis. APMIS 2007;115:809-13. 